Journal of Information and Communications Engineering (JICE), 6 (2): 391-397, December 31, 2020

e-ISSN: 2432-5465, p-ISSN: 2186-9162

Applied Science and Computer Science Publications

\title{
Assessing of frequency dynamics of EEG signals in a visualization experiment related to crime deterrence
}

\author{
Rafael D.E.H. Castro*, Martha Peña-Sarmiento**, Ervyn H. Norza C.***, Camilo A. Sanchez*, Erick Gillen*, \\ Yeizon A. Duarte***, Luis O. Jimenez A**
}

\begin{abstract}
The purpose of this document is to assess Electroencephalographic (EEG) signal frequency dynamics in visual stimuli related to crime deterrence from an inexpensive device. The signals were acquired from 4 participants, with an EMOTIV EPOC 14 channel EEG device, while visual stimuli (deterrence and neutral) were presented, also an eye-tracking device was used to follow the participants visual path through the images, the experimental design was developed in the Paradigm software and the signal processing in Python using MNE for the EEG data analysis. Methods: The signal pass by a preprocessing which includes filtering, denoising and ICA object rejection, then the Global Field Power (GFP) is calculated to track the temporal dynamics of frequency bands theta, alpha, beta and gamma, finally differential GFP for theta and alpha bands is calculated and maximal temporal frequency responses are represented. The process applied shows dynamic characteristics of frequency bands and allows maximal localization of its responses.
\end{abstract}

Keywords: Electroencephalography; Global Field Power; Visual; Signals; Neuroscience

\section{INTRODUCTION}

$\mathrm{T}$ his work is part of a larger research on criminal behavior which purpose is to identify the relationship between threat communication and Perceived deterrence. Deterring individuals from participating in illegal activity is the main purpose of both criminal justice public policy and several parts of criminal-justice system [1]. Deterrence has been conceptualized from a criminological or an economic tradition both with core concepts embedded in theories of deterrence [1]: the principles of certainty, severity, and celerity on punishment as well as the concept of expected utility [2]. Nevertheless, considering that every effective attempt to change people behavior depends on public's perceptions of the certainty, severity, and celerity of punishment [3]; perceived deterrence becomes a critical psychological variable at any explanation model. Measuring perceived deterrence and relating it to expected neurological reactions to threat communication is a challenge in this research.

Measurement tradition in experimental psychology has used reaction time, correctness of responses [4] forcedchoice tasks [5], and self-report formats [6] to obtain information on behavior and cognitive processes.
Although, those measures reveal part of our psychological functioning, they have important limitations that neurophysiological correlates have proven to overcome [7]. Nowadays, researchers tend to use behavioral and neurophysiological measures as a complement [8]. Threat communication mainly based on an arrangement of messages and emotional facial expressions, alters cognitive processing and EEG signals allow us to track in real time those changes in the brain. Based on waves patterns observed and other data as eye tracking movements it is possible to discriminate differential effect of exposition to criminal versus non-criminal groups.

Deterrence has been an influential approach into Police worldwide in last two decades [9][10]. Currently, Police force assumes that deterring effect could be even higher if offender's perception on punishment certainty increases. Similar effect is expected in people at risk to behave illegally [11][12] or citizens in general; results which allows to police department preserving its size, while it focusses on communications strategies that change subjective probability of punishment certainty [13]. This research aims at testing that assumption from the perspective of Neuro-criminology, a multidisciplinary field where neurosciences,

Corresponding Authors: Rafael Castro* E-mail: rafael.castro-r@uniminuto.edu.co , Martha Peña-Sarmiento E-mail: mrpena@ucatolica.edu.co ** and Ervyn Norza***E-mail: ervyn.norza@correo.policia.gov.co *Minuto de Dios University, ** Catholic University of Colombia, *** National Police Crime Observatory 
criminology and engineering converge [14]; as a Proactive policing's effort [15] to improve our understanding on how deterrence works.

Electroencephalographic (EEG) signals are interesting objects to the brain's study, since the first evidence of electric current in the brain by Caton (1875), and the first EEG record in humans by Berger (1929) arise a race to understand its characteristics related to pathologies, emotions, cognition, language, visual imagery and even ways to use them as control signals [16]. In particular for cognition various relationships has been studied for certain frequency bands theta $(\theta)$ [4 $\mathrm{Hz}-7 \mathrm{~Hz}]$, alpha $(\alpha)[7 \mathrm{~Hz}-13 \mathrm{~Hz}]$, beta $(\beta)[13 \mathrm{~Hz}-$ $30 \mathrm{~Hz}]$ and gamma $(\gamma)[30 \mathrm{~Hz}-100 \mathrm{~Hz}]$, as an example Klimesch in [17] where $\alpha$ and $\theta$ ranges and the amount of EEG power in their magnitudes -in an inverse relationship- is associated to cognitive and memory performance; or Van der Hiele who shows that an increase in $\theta$ relative power and a decrease in $\alpha$ reactivity, relates to worse neuropsychological test performance in several cognitive domains [18]. Zaepffel who observed $\beta$ power decrease during movement preparation and execution of grasping and increment around the start signal of the movement ordered [19]. The neural basis of the relationships between grouping, attention, memory and awareness points to $\gamma$ activity bended to physiological properties of neurons and cognitive task synchronization [20]. Through brain intrinsic rhythms, typically in the $8 \mathrm{~Hz}-$ $40 \mathrm{~Hz}$ frequency, some of them related to periodic activity of peripheral sensor and effector organs [21] those relationships show how an understanding of frequency dynamics on the EEG signals can be significative.

The approach here pretends to obtain the activity dynamics for certain frequency bands, it is achieved by means of the Global Field Power (GFP), which corresponds to the spatial standard deviation of the complete electrode field and usually is used to quantify the activity magnitude[22], applied to the filtered signal in the specific frequency bands, with a limit of $45 \mathrm{~Hz}$ due to the EEG device characteristics

\section{INSTRUMENTS AND SOFTWARE}

\section{A. Instruments}

For the acquisition of electroencephalographic signals, a commercial and low-cost equipment, the Epoc, from the Emotiv company has been used. This device consists of 14 channels distributed approximately as in the modified 10-20 system [8] with electrodes in AF3, F7, F3, FC5, T7, P7, O1, O2, P8, T8, FC6, F4, F8, AF4; The equipment has an acquisition rate of $128 \mathrm{~d} / \mathrm{s}$ and a resolution of 14 bits with a Least Significant Bit value of $51 \mu \mathrm{V}$, plus 2 default references channels CMS (P3) as an absolute voltage reference and DRL (P4) as a feedback cancellation system to emit the reference level in the common mode of body potential [23]. The communication from the device to the acquisition computer is by means of wireless connectivity, Bluethoot. The Emotiv-Testbench software allows to save the EEG data in an EDF format, which can be converted in a CSV file.

For the eye tracking data, the equipment used was the Mobile Eye-5 eye tracking glasses, from ASL. The Mobile Eye-5 has an acquisition rate of $30 \mathrm{~d} / \mathrm{s}$ and 60 $\mathrm{d} / \mathrm{s}$, it can be used both as wireless and PC-direct connection in its network interface, as an independent device the data is stored in a SD card, [24]. The device counts with 2 cameras, one for the eye with a 3 reflective pupil-cornea references, from a monocular lent in the right eye; other camera for the scene, with visual range of 60 degrees horizontal, 40 degrees vertical and 0.5 degrees of accuracy, [25]. The device returns: XY coordinates of the pupil and referenced scene calibration pupil position, the pupil diameter, time and marker stamps.

\section{B. Software}

Testbech, the proprietary software from Emotiv company to adquire and save the EEG data, the result is presented in an EDF file.

- MNE-PYTHON, a specialized software for MEG and EEG signal analysis, [26], along with various PYTHON packages are used for the EEG data processing and visualization.

- Paradigm software for the stimuli presentation, plus two libraries: Paradigm elements for ports, and Paradigm elements for ASL in order to send synchronous stimuli markers.

\section{EXPERIMENTAL SETUP}

The physical configuration of the system is shown in the figure 1. There are two laptops, one EEG device, one Eye-Tracker device and one switch. In laptop Pres the Paradigm Software is installed to the stimuli presentation and markers sending to both EyeTrack EEG devices due to synchronization of the stimuli. The ASL device is connected interfacing the Eye Tracking 
glasses. The EEG laptop has the testbench software for the acquisition of EEG data, the Emotiv Epoc connects to it via Bluetooth. A switch element stablish a network connection for all the systems.

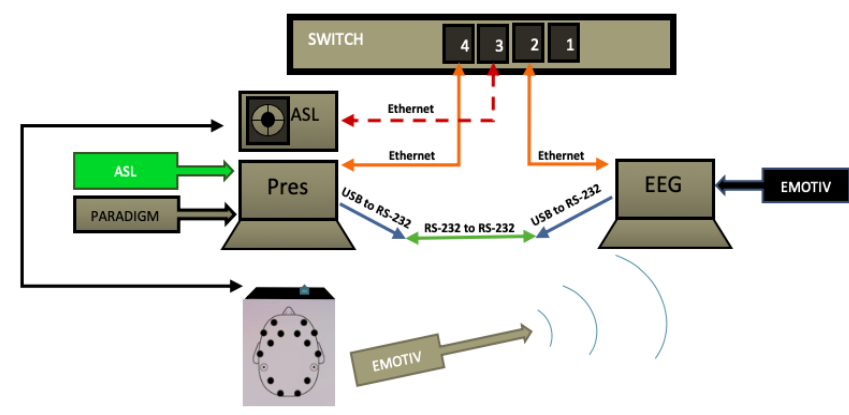

Fig. 1. Setup for the stimuli presentation, EEG and Eye-Tracking data acquisition.

The experimental imaging task begins with a neutral image followed by a visual deterrent, this happens repeatedly, with 30 different marked visual deterrents, each with 6 seconds duration; the set of images is randomized for each participant in the Figure 2 there is an example of the used material sequence. Minimum movement of the participants is required and measured by means of the EEG accelerometers an gyros.

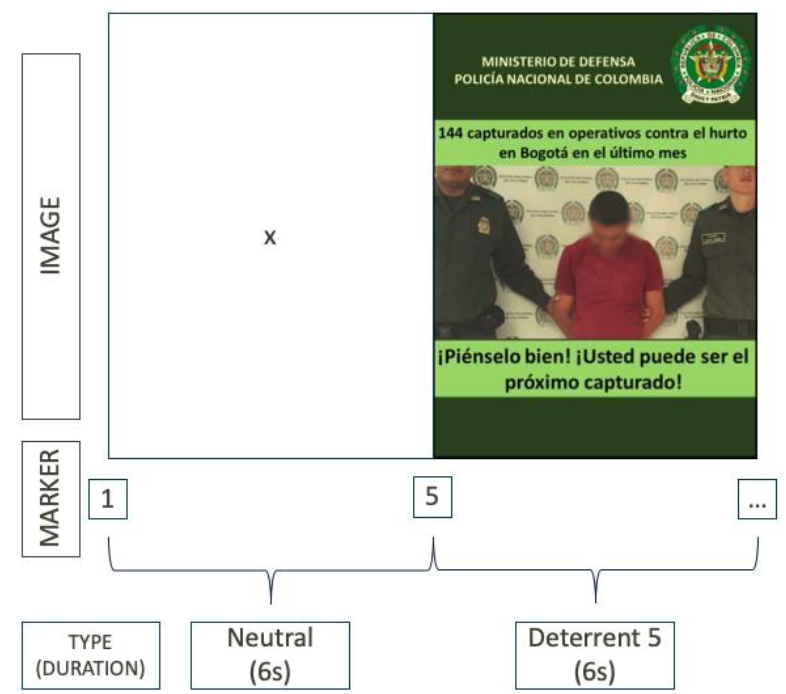

Fig. 2. Samples of neutral and deterrent visuals, where each experimental stimuli has a marker and lasts 6 seconds.

\section{PREPROCESING}

Acquired signals of EEG usually contains other sources which are not related to brain behavior such as related electromyography (EMG), electrocardiography (ECG), electrooculography (EOG) signals so the methodology proposed for the preprocessing includes the segmentation of data per individual and the stimuli event space, by associating the event markers to the EEG raw signal; Digital pass band filtering at $2.5 \mathrm{~Hz}$ and $50 \mathrm{~Hz}$ for low driving and high frequencies noise of electric origin removal, a finite impulse response filter (FIR) is used due to easier shift compensation and control [27] in the EEG signal, it is useful when the base signal has finite duration, as EEG is related to finite neuron potential combination. The power spectral density of the filtered signals is shown in Figure 3.

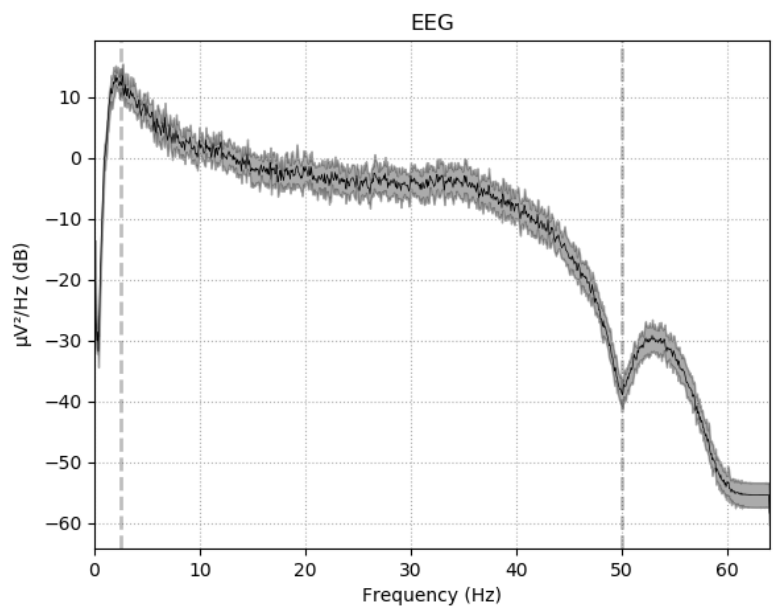

Fig. 3. Power spectral density plot of passband filtered signal between $2.5 \mathrm{~Hz}$ and $50 \mathrm{~Hz}$.

Artifact removal by infomax independent component analysis (ICA) extraction (EOG, EMG, ECG) is applied due to its reliability in non-EEG artifact rejection [28][29].

ICA in signal processing is a technique to find a matrix $\mathrm{W}$ and a vector $\mathrm{w}$ for the signal transformation $\mathrm{x}$ where the transformation $s=\left[s_{1, \ldots, s} s_{n}\right]^{T}$ has statistically independent elements $s_{i}$ in

$$
s=W_{x}+w
$$

[30][31]. Usually $\mathrm{x}$ comes from a source collection where all the signals interact between them, the ICA can approximate the value of the independent sources, including those no related or noisy.

Figure 3 shows the 14 components obtained; the components closely related to EOG and EMG are selected as noisy references for recalculating the signal, the criteria, for maintaining the most of EEG 
information, includes bad channel and lapse rejection (just if needed) [32], for the 4 participants cases ICA was sufficient for EOG and EMG rejection. The Figure 4 shows a sample of the ICA maps for the 14 components and Figure 5 shows a EOG noise component in this case ICA001 and average result signal.

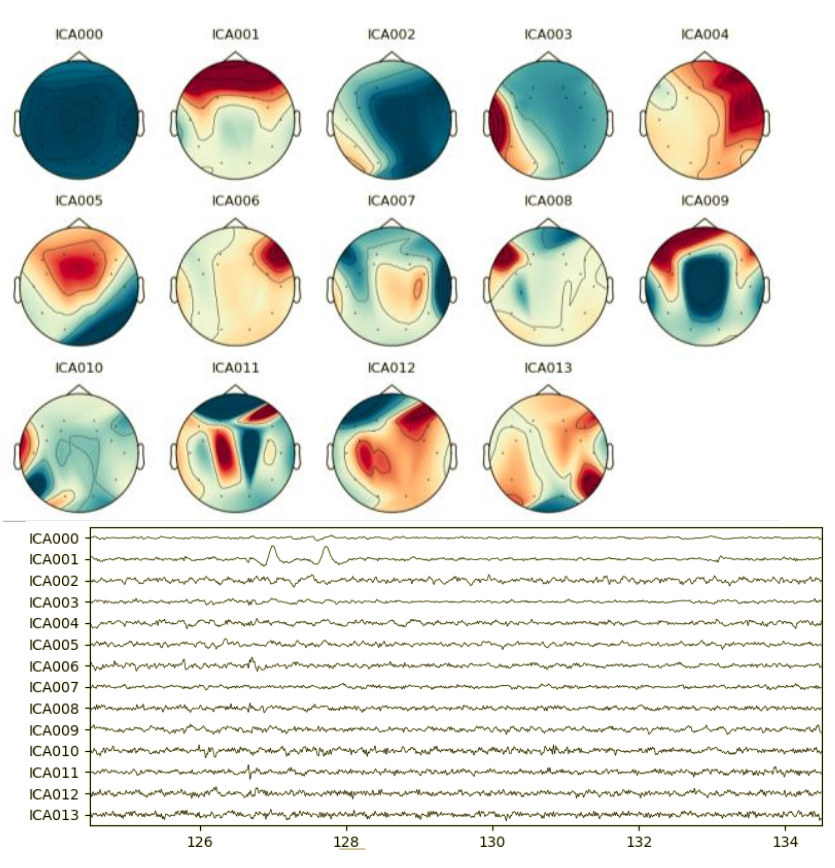

Fig. 4. Sample of ICA components for EEG data, topomaps (up) and time projection of isolated components (down).
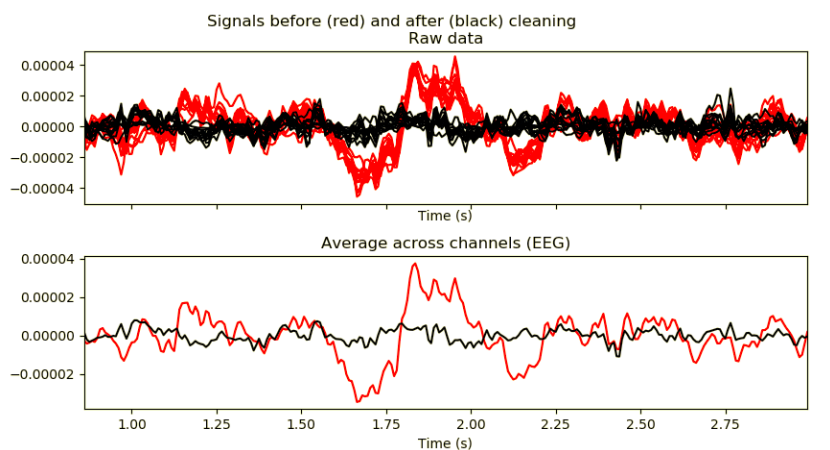

Fig. 5. ICA001, a component with related EOG noise (up), ICA EOG removed EEG average signal red before, black after (down).

After preprocessing the signal should contain EEG the most.

\section{GLOBAL FIELD POWER (GFP) CALCULATION}

To obtain specific band frequency dynamics for bands $\theta$ [ $4 \mathrm{~Hz}-7 \mathrm{~Hz}], \alpha[7 \mathrm{~Hz}-13 \mathrm{~Hz}], \beta[13 \mathrm{~Hz}-30 \mathrm{~Hz}]$ and $\gamma[30 \mathrm{~Hz}-45 \mathrm{~Hz}]$, a FIR passband filter is applied, next the GFP is calculated as (1) in [33] and [34] over the complete electrode field for the filtered bands:

$$
\mathrm{GFP}_{\mathrm{B}}=\frac{\sum_{k=0}^{N} x_{N}^{2}}{N}
$$

Where GFP_B is the GFP for the band $\mathrm{B}, \mathrm{N}$ is the number of electrodes, in the Figure 6, band frequency GFPs plots of the 4 bands for neutral deterrent stimuli.

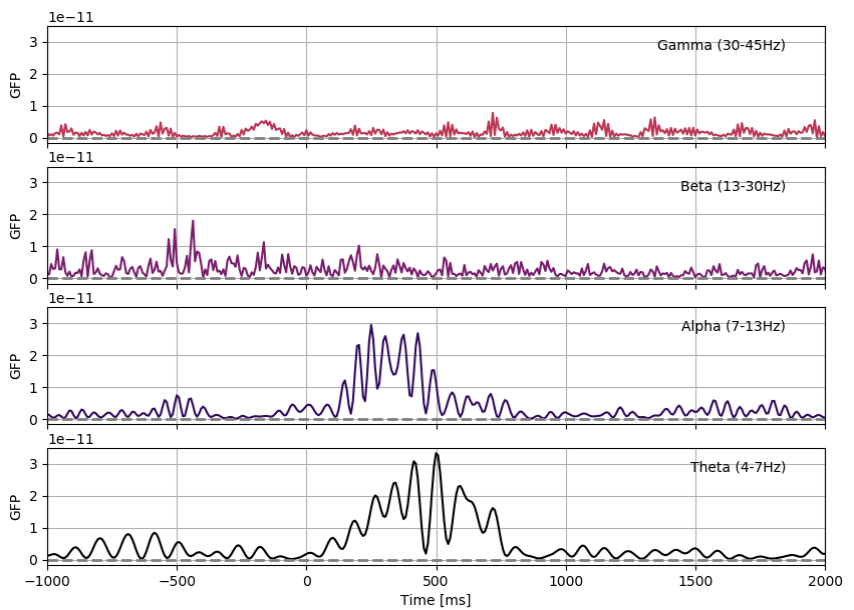

Fig. 6. GFP for $\alpha, \beta, \theta$ and $\gamma$ bands, the zero position indicates the deterrent image start, waves from participant 2 .

Different response are observed for each participant, for example in Figure 1 participant 2 shows a stronger response of $\theta$ and $\alpha$ waves, but for participant 4, in Fig. 7, $\alpha, \beta$ are stronger.

Dynamics for the frequency bands are achieved but for instance some relationship between those dynamics can be important for psychological evaluation of the response to a deterrent stimuli, as such a differential of $\theta$ and $\alpha$ waves, cognition related [17][18] is presented in Figure 8, as some peaks of the differential field obtained. 


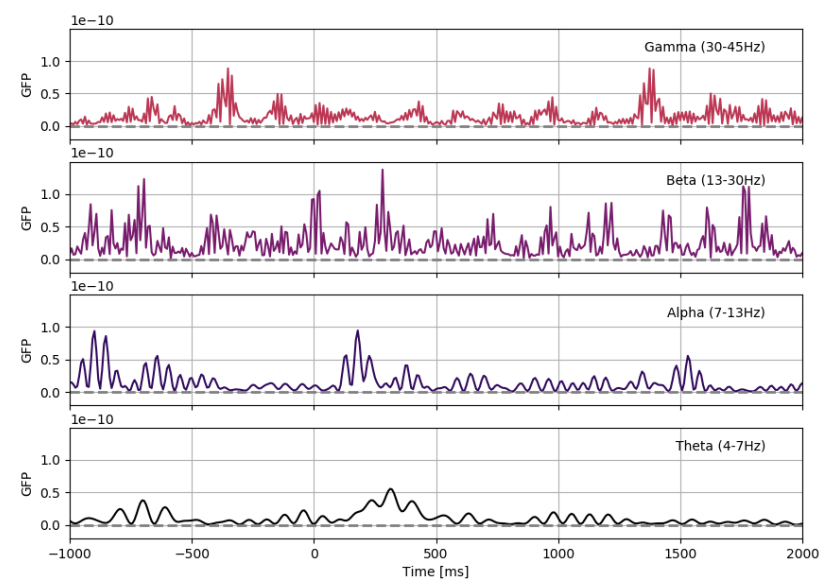

Fig. 7. GFP for $\alpha, \beta, \theta$ and $\gamma$ bands, the zero position indicates the deterrent image start, waves from participant 4.

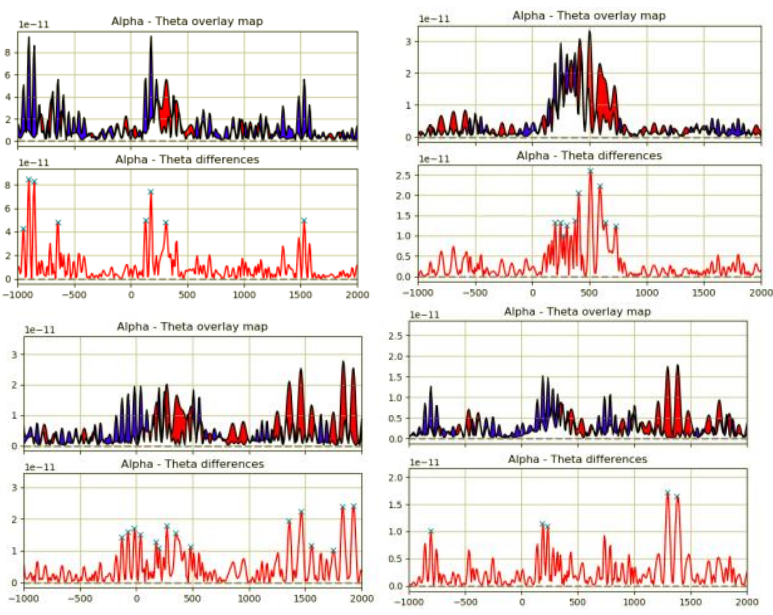

Fig. 8. GFP differential graph of $\theta$ and $\alpha$ bands for the 4 participants, the red fill indicates when $\theta$ instantaneous power is greater than $\alpha$ 's (up); Absolut difference graph of $\theta$ and $\alpha$ bands, the peaks indicate local maxima in the differential curve.

Those same peaks can be observed over the raw signal, with their respective topomaps in the Figure 9. A forward study will compare cognition activity related deterrence while deterrent stimuli is presented.

The process to follow could be represented like in Figure 10, where preprocessing, frequency band segmentation, event segmentation, GFP calculation and GFP differential applications achieve the proposed objective for assessing frequency dynamics of EEG signals.

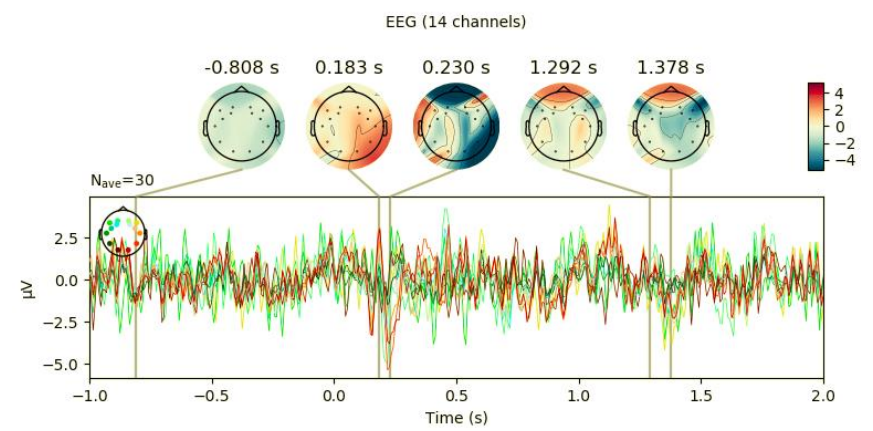

Fig. 9. GFP $\alpha, \theta$ differential dynamic peaks topomaps over the EEG signal.

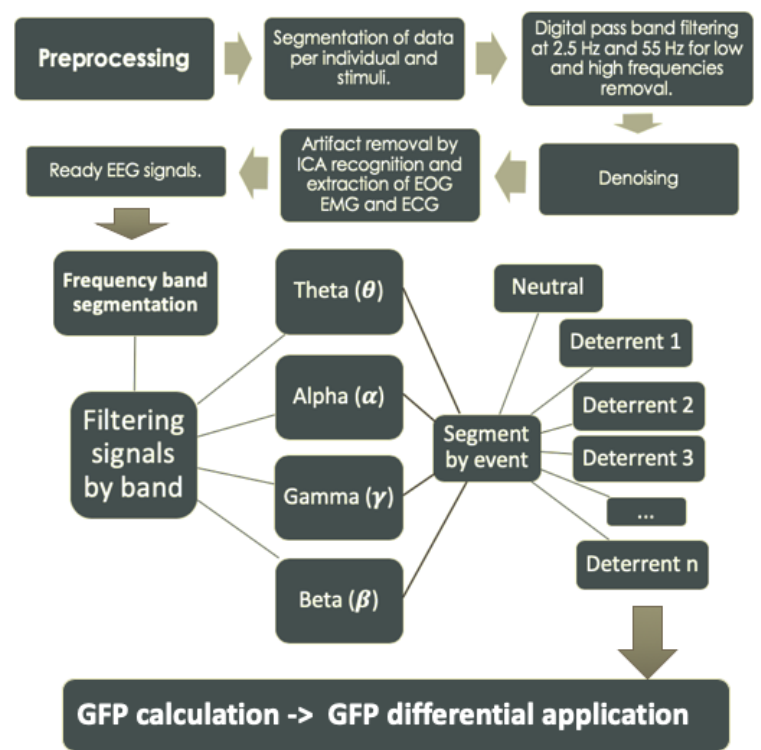

Fig. 10. Process implemented.

\section{CONCLUSIONS}

The developed process achieves showing important characteristics of frequency bands temporal dynamics introduced in visual stimuli, by means of the GFP and operable comparitions of GFPs. The methodology to assess frequency dynamics is achieved and can be applied to study some characteristics of frequency bands over time, event taking into account differential dynamics important for cognition or others brain functions. Critical steps are presented in the EEG preprocessing, more specific in the ICA application due to criteria application for reject noisy component without compromising the brain signal activity. 


\section{ACKNOWLEDGEMENTS}

We would like to acknowledge the Minuto de Dios University, the Catholic University of Colombia and The Crime Observatory of The National Police of Colombia for the support of the project.

\section{REFERENCES}

[1] Chalfin, A., \& McCrary, J. (2017). Criminal deterrence: A review of the literature. Journal of Economic Literature, 55(1), 5-48. doi: 10.1257/jel.20141147

[2] Apel, R. (2013). Sanctions, perceptions, and crime: Implications for criminal deterrence. Journal of quantitative criminology, 29(1), 67-101. doi: 10.1007/s10940-012- 9170-1

[3] Paternoster, R. (2018). Perceptual deterrence theory. In Nagin, D., Cullen, F., \& Jonson, C. (Eds.), Deterrence, Choice, and Crime, Volume 23 (pp. 81-106). New York, NY: Routledge Taylor \& Francis. doi: $10.4324 / 9781351112710$

[4] Luu, P., Poulsen, C., \& Tucker, D. M. (2009). Neurophysiological measures of brain activity: Going from the scalp to the brain. In International Conference on Foundations of Augmented Cognition (pp. 488-494). Berlin: Springer.

[5] Ratcliff, R., Voskuilen, C., \& Teodorescu, A. (2018). Modeling 2-alternative forced- choice tasks: Accounting for both magnitude and difference effects. Cognitive psychology, 103, 1-22. doi: 10.1016/j.cogpsych.2018.02.002

[6] Fryer, L. K., \& Nakao, K. (2020). The future of survey self-report: An experiment contrasting Likert, VAS, slide, and swipe touch interfaces. Frontline Learning Research, 8(3), 10-25. doi: 10.14786/flr.v8i2.502

[7] Dahlstrom -Hakki, I., Asbell -Clarke, J., \& Rowe, E. (2019). Showing is knowing: The potential and challenges of using neurocognitive measures of implicit learning in the classroom. Mind, Brain, and Education, 13(1), 30-40. doi: $\quad 10.1111 / \mathrm{mbe} .12177$

[8] Tinga, A. M., De Back, T. T., \& Louwerse, M. M. (2019). Non-invasive neurophysiological measures of learning: A meta-analysis. Neuroscience \& $\begin{array}{lll}\text { Biobehavioral } & \text { Reviews, } 99,89 .\end{array}$ doi:10.1016/j.neubiorev.2019.02.001

[9] Sherman, L. W. (2013). The Rise of Evidence-Based Policing: Targeting, Testing, and Tracking. Crime and Justice, 42(1), 377-451. doi:10.1086/670819

[10] Weisburd, D., \& Braga, A. A. (2019). Police Innovation: Contrasting Perspectives. Cambridge University Press

[11] Braga, A. A. (2001). The Effects of Hot Spots Policing on Crime. The ANNALS of the American Academy of Political and Social Science, 578(1), 104-125. doi:10.1177/000271620157800107

[12] Nagin, D. S. (2013). Deterrence in the Twenty-First Century. Crime and Justice, 42(1), 199-263. doi:10.1086/670398

[13] Nussio, E., \& Norza, E. (2018). Deterring delinquents with information. Evidence from a randomized poster campaign in Bogotá. PLOS ONE, 13(7), e0200593. doi:10.1371/journal.pone.0200593

[14] National Academies of Sciences, Engineering, and Medicine. (Ed.). (2018). Proactive Policing: Effects on Crime and Communities. National Academies Press

[15] Glenn, A. L., \& Raine, A. (2014). Neurocriminology: Implications for the punishment, prediction and prevention of criminal behaviour. Nature Reviews Neuroscience, 15(1), 54-63. doi:10.1038/nrn3640

[16] Sanei, Saeid, and Jonathon A. Chambers. EEG signal processing. John Wiley \& Sons, 2013

[17] Klimesch, Wolfgang. EEG alpha and theta oscillations reflect cognitive and memory performance: a review and analysis. Brain research reviews, 29(2-3), pp. 169-195

[18] Van der Hiele, K., Vein, A. A., Reijntjes, R. H. A. M., Westendorp, R. G. J., Bollen, E. L. E. M., Van Buchem, M. A., ... \& Middelkoop, H. A. M. (2007). EEG correlates in the spectrum of cognitive decline. Clinical neurophysiology, 118(9), 1931-1939

[19] Zaepffel, M., Trachel, R., Kilavik, B. E., \& Brochier, T. (2013). Modulations of EEG beta power during planning and execution of grasping movements. PloS one, 8(3), e60060. https://doi.org/10.1371/journal.pone.0060060

[20] Tallon-Baudry, C. (2009). The roles of gamma-band oscillatory synchrony in human visual cognition. Front Biosci, 14, 321-332

[21] Hari, R., \& Salmelin, R. (1997). Human cortical oscillations: a neuromagnetic view through the skull. Trends in neurosciences, 20(1), 44-49

[22] Skrandies, W. (1990). Global field power and topographic similarity. Brain topography, 3(1), 137-141

[23] Deuschl, G., \& Eisen, A. (Eds.). (1999). Recommendations for the practice of clinical neurophysiology: guidelines of the International Federation of Clinical Neurophysiology (No. 52). Elsevier Health Sciences

[24] Duvinage, M., Castermans, T., Dutoit, T., Petieau, M., Hoellinger, T., Saedeleer, C. D. \& Cheron, G. (2012). A P300-based quantitative comparison between the Emotiv Epoc headset and a medical EEG device. Biomedical Engineering, 765(1), 2012-2764.

[25] Applied Science Laboratories, "ASL Mobile Eye-5 Glasses", Est-kl.com, 2016. [Online]. Available: https://est-kl.com/images/PDF/ASL/Mobile\%20Eye5\%20Brochure.pdf. [Accessed: 03- Apr- 2020]

[26] Applied Science Laboratories, "ASL Mobile Eye-5 Glasses, Specifications", Est-kl.com, 2016. [Online]. Available: https://estkl.com/images/PDF/ASL/Mobile\%20Eye $\% 205 \% 20$ Spec ifications.pdf [Accessed: 04- Apr- 2020]

[27] Widmann, Andreas, and Erich Schröger. "Filter effects and filter artifacts in the analysis of electrophysiological data." Frontiers in psychology 3, 2012, pp. 233, doi: 10.3389/fpsyg.2012.00233.

[28] Zhaoiun Xue, Jia Li, Song Li and Baikun Wan, "Using ICA to remove eve blink and power line artifacts in EEG," First International Conference on Innovative Computing, Information and Control - Volume I (ICICIC'06), Beiiing, 2006, pp. 107-110, doi: 10.1109/ICICIC.2006.543.

[29] Dimigen, Olaf. "Optimizing the ICA-based removal of ocular EEG artifacts from free viewing experiments." 
Neuroimage 207, 2020, 116117. Doi: 10.1016/j.neuroimage.2019.116117

[30] Makeig, S., Bell, A. J., Jung, T. P., \& Sejnowski, T. J. (1996). Independent component analysis of electroencephalographic data. In Advances in neural information processing systems (pp. 145-151).

[31] Langlois, D., Chartier, S., \& Gosselin, D. (2010). An introduction to independent component analysis: InfoMax and FastICA algorithms. Tutorials in Quantitative Methods for Psychology, 6(1), 31-38.

[32] Picton, Terence. W., et al. Guidelines for using human event - related potentials to study cognition: Recording standards and publication criteria. Psychophysiology, 2000, vol. 37, no 2, pp. 127-152.

[33] ENGEMANN, Denis A.; GRAMFORT, Alexandre. Automated model selection in covariance estimation and spatial whitening of MEG and EEG signals. NeuroImage, 2015, vol. 108, pp. 328-342.

[34] D. Engemann and S. Appelhoff, "Explore event-related dynamics for specific frequency bands - MNE 0.20.7 documentation", Mne.tools. [Online]. Available: https://mne.tools/stable/auto_examples/time_frequency/p lot_time_frequency_global_field_power.html?highlight= gfp. [Accessed: 06- May- 2020] 